\title{
Effets des aménagements anciens
}

\author{
Monique Dacharry \\ Professeur à l'Université des Sciences et Techniques de Lille
}

Maintes découvertes archéologiques en milieux fluviaux, à l'occasion de travaux effectués aujourd'hui dans les plaines alluviales, attestent l'ancienneté de l'aménagement des fleuves et des rivières. Ainsi les fouilles archéologiques récentes, alentour des années 1980 , à l'intérieur d'un méandre de la Seine au site mésolithique de Noyen situé à vingt kilomètres en amont de Montereau, ont prouvé la permanence de l'occupation des lieux dès le septième millénaire avant notre ère ; elles ont précisé une orientation des activités halieutiques au rythme des saisons ; elles ont mis à jour les témoins d'une industrie diversifiée : débitage lithique, céramique, nasse, pirogue... ; elles ont même révélé l'existence d'ouvrages dans le lit mineur du fleuve, sortes de palissades édifiées à plusieurs reprises pour renforcer le rôle de barrage joué par un paléochenal en cours de colmatage. De temps immémorial, les hommes ont utilisé les cours d'eau et, comme il n'est guère de partie du globe où la distribution naturelle des eaux courantes au long des saisons est si bien réglée qu'elle n'appelle quelque amélioration artificielle, la "domestication " de l'eau se manifeste de façon universelle. Elle a même, parfois, fait naître des ouvrages qui restent caractéristiques d'une civilisation, tels ces réseaux d'eau potable, plurimillénaires, du MoyenOrient, d'Inde ou de Chine. Ailleurs, digues, canaux d'irrigation, fossés, déversoirs, roues hydrauliques font partie intégrante de paysages actuels, ce qui n'est pas le moindre effet de leur construction. Ainsi en est-il, par exemple, des fossés ceinturant de vieilles villes fortifiées, des « wateringues » de la plaine maritime du Nord de la France ou des "levées" du Val de Loire, étudiées par R. Dıon : "Au bord d'une Loire parvenue à la plénitude de sa puissance, ces signes visibles d'un travail collectif plusieurs fois sécu- laire ajoutent à la majesté des perspectives fluviales. Ils offrent à un esprit curieux du passé cet aliment de choix qu'est le document prenant forme de trait de paysage. "

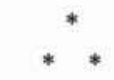

A chaque époque et sous toutes les latitudes, aux besoins fondamentaux pour la boisson, la circulation, l'arrosage des terres et la force motrice s'ajoutent, souvent, la lutte contre la surabondance des eaux, leur pénurie ou leur pollution. Ces besoins divers sont ressentis inégalement selon les conditions naturelles locales et la pression d'une demande mesurée par le nombre de la population, ses activités, son degré d'évolution socio-économique. Ils sont satisfaits par les moyens techniques connus à l'époque considérée et en fonction des possibilités financières. Cet ensemble de conditions tenant aux besoins et aux moyens conduit à distinguer deux grandes phases ou états " anciens" de civilisation hydraulique.

La première période, très longue, est celle des faibles moyens techniques, qui dure depuis les temps préhistoriques jusqu'à ce que l'homme ait à sa disposition des machines motorisées. C'est l'ère à dominante "agricole » où les sociétés rurales, pour leur quête de l'eau, tirent parti avec ingéniosité des moindres possibilités qu'offrent les configurations naturelles de l'espace: dénivellations parfois infimes, fonds humides pour créer des petits étangs artificiels et installer des pêcheries, versants montagneux savamment irrigués, courants d'eau jalonnés de moulins, rivières navigables et flottables... C'est le temps des «turcies" et "levées » contre les crues dans les plaines allu-

\section{Effects of old developments}

A deeper and deeper knowledge of processes in all environmental fields enables us to better appreciate the direct or indirect, slow or rapid, beneficial or harmful consequences of river developments and thus enables us to take these consequences into account when planning projects or carrying out large hydraulic works. In the criticisms often made against new developments, it is often alleged that lighter techniques or practises, no longer used now, would have been more successful in providing a satisfactory balance between group interests and water requirements. Indeed, there may be lessons to be learned from old developments while still keeping in mind the scale of needs and technical possibilities. 
viales, des dérivations de cours d'eau à fins défensives pour ceinturer les villes du Moyen-Age, ou pour distribuer l'eau aux citadins et artisans dans ce que A. GUILLERmE a justement appelé les "petites Venises». Comme la prospérité économique ne va pas sans les «chemins d'eau ", les grandes rivières mais aussi de petits cours d'eau, aujourd'hui simples ruisseaux, sont creusés et aménagés pour porter des bateaux. Un exemple très démonstratif est donné par la rivière d'Etampes, la Juine, objet d'une " canalisation " systématique au $\mathrm{XVI}^{\mathrm{e}}$ siècle, avec rassemblement des eaux dans un chenal unique, consolidation et exhaussement des rives, installation d'écluses pour diminuer la pente, ralentir l'écoulement et prolonger la période où la rivière est utilisable. Il semble qu'à l'époque d'Henri IV, la rivière, en dépit de ses apparences actuelles, fut une voie d'eau essentielle à l'économie parisienne. Le cas de cette rivière d'Etampes n'est pas isolé dans l'ancienne économie de l'Europe occidentale.

Dans cette utilisation ancienne et diversifiée de l'eau, quelques caractères sont dominants :

- la faiblesse des moyens techniques et la prolifération de minuscules ouvrages (une trentaine de moulins, par exemple, de Royat à Clermont-Ferrand, sur vingt kilomètres, le long de la Tiretaine);

- la finalité unique des aménagements : ainsi, dans le Val de Loire, certaines levées servent les besoins de l'agriculture riveraine alors que d'autres, un peu plus loin, ont été édifiées par les bourgeois des villes pour améliorer la navigation ;

- la multitude de litiges, si limités qu'aient été ces ouvrages, en raison du grand nombre de propriétés et servitudes affectées. Les grands cataclysmes qui réduisent les oppositions entre propriétaires, tous victimes, appellent l'intervention du plus haut arbitre et de la plus puissante autorité. Toujours dans le bassin de la Loire, en 1571, considérant les dommages infligés aux riverains du fait des débordements répétés du fleuve, le Conseil du Roi établit un Surintendant des Torcies et Levées pour ordonner "l'assiette, establissement et entretènement" desdits ouvrages sur toute la longueur du cours de la Loire.

Il n'est pas possible de fixer un terme précis à cette période parce que nombre de ses caractères se maintiennent plus ou moins après les grands bouleversements introduits par ce qui est entré dans le vocabulaire des historiens sous le terme de "révolution industrielle».

La seconde période des aménagements " anciens » débute, en effet, avec l'apparition du machinisme qui prend vite des dimensions telles que les structures économiques, sociales, démographiques en sont transformées fondamentalement. Cette période dure, en gros, deux siècles, de 1750 à 1950 . Le développement de l'industrie, l'urbanisation, l'augmentation de la population (en France, 18 millions en 1745 et 31 millions au recensement de 1821) provoquent une dilatation et une diversification accélérées des besoins en eau.

Successivement ou tout ensemble, les idées physiocratiques, les progrès techniques, l'augmentation des moyens financiers, l'organisation administrative favorisent la science hydraulique qui multiplie ses applications dans des domaines particuliers :
- l'amélioration de l'agriculture avec, notamment, l'introduction du drainage souterrain et l'œuvre de bonification des marais;

- la navigation intérieure ;

- l'assainissement urbain et l'hygiène (le seul nom de Belgrand étant particulièrement évocateur);

— enfin l'énergie hydroélectrique...

Les Edits d'Henri IV de 1599 et 1607 sont, en France, les premières connues des dispositions du pouvoir central sur le dessèchement des marais. Les œuvres de bonification dans le Bas-Dauphiné s'étendent de 1668 à 1814. La technique du drainage souterrain sera appliquée à partir de 1850 dans le Boulonnais.

C'est à partir de 1750 et en moins de cinquante ans qu'est réalisé en Angleterre, pour les besoins accrus des cités industrielles, un véritable réseau de voies navigables avec construction de canaux.

En France, la "route d'eau» ligérienne, malgré une médiocre navigabilité, marque des points jusqu'en 1830 avec la mise en service de bateaux à vapeur et roues à aubes, qui ne résisteront pas cependant après 1850 à la concurrence des chemins de fer. L'aménagement de la Seine et la coordination de canaux de jonction et des rivières convergeant vers Paris, s'échelonnent depuis le début du $\mathrm{XIX}^{\mathrm{e}}$ siècle jusqu'au milieu du $\mathrm{Xx}^{\mathrm{e}}$ siècle.

La production d'énergie hydroélectrique, où la France a eu un rôle pionnier, fut d'abord limitée aux vallées montagnardes qui se prêtaient à l'utilisation directe des cours d'eau abondants. Les réservoirs régulateurs ne pouvaient pas apparaître avant que le transport de l'électricité fut techniquement possible (1895). Mais c'est une condition juridique qui a été finalement décisive. En France, la loi du 16 octobre 1919 sur l'utilisation de l'énergie électrique a ajouté ou substitué d'autres principes à ceux qui découlaient de la législation antérieure, sur la jouissance privative des riverains notamment. L'ère des grands barrages modernes commence pratiquement avec le barrage d'Eguzon qui date de 1926.

Les aménagements "anciens » cessent à partir du moment où les projets acquièrent une ampleur qui embrasse la gestion des richesses en eau, non seulement des rivières, mais de leur bassin versant, et cela en harmonie avec l'aménagement du territoire. Seul l'Etat peut prendre une vue globale du bien commun national et même quelquefois international, et disposer des moyens nécessaires à la mise en œuvre des projets globaux au long des années. La création, en 1933, de la Tennesse Valley Authority en est une première illustration.

Les premiers effets immédiats des aménagements sont contemporains de ces aménagements mêmes et ces effets seront plus ou moins marqués selon les dimensions de l'ouvrage et la sévérité des atteintes à l'état naturel. 
Un exemple d'aménagement doux est donné par le travail répété auquel s'appliquaient encore les paysans riverains de la rivière Diyalah, non loin de Bagdad, sous la domination turque. Quand les crues de la rivière remplissaient les canaux d'irrigation, les paysans construisaient à leur entrée des barrages de terre et de broussailles qui retenaient l'eau dont le niveau baissait dans la rivière durant la saison des basses eaux. Au retour de la saison des hautes eaux, la première crue balayait les très fragiles barrages. Cet aménagement, à l'évidence, ne causait qu'une atteinte légère, et pour ainsi dire effaçable, aux équilibres naturels.

Ce sont encore des ouvrages sans grande ambition mais cependant plus lourds de conséquence que ceux qui ont été construits sur le Rhin entre Bâle et la frontière hollandaise à la fin du XVIII siècle. Fruits d'initiatives isolées, efficaces seulement sur de brefs parcours et contraires à une politique de modernisation globale, ils n'ont pas créé une voie d'eau commode et, vers 1850, l'Ingénieur en chef de la Navigation en dénonçait les défauts. L'unité d'action des riverains s'est faite pour canaliser le fleuve mais les travaux achevés en 1860 faillirent manquer leur but. Le premier effet fut d'augmenter la force du courant et, jusqu'à l'apparition de la navigation à vapeur, le trafic de Strasbourg en subit un grave dommage. L'exemple montre que, pour être efficaces, les projets doivent atteindre une certaine échelle et ne sauraient ignorer aucune de leurs conséquences. Le premier résultat de la canalisation n'a pas été d'améliorer la navigation, but visé, mais de mettre définitivement les riverains à l'abri des crues.

Autre exemple de la nécessité de projets d'ensemble, les résultats décevants des travaux entrepris au XVIII ${ }^{\mathrm{c}}$ siècle pour protéger Perpignan contre les crues de la Têt. Après trente ans de travaux successifs et en différentes parties du lit, les ingénieurs comprirent l'insuffisance des aménagements ponctuels et la nécessité d'un projet cohérent portant sur tout le bassin.

La prévision des conséquences entrainées par la modification de l'écoulement a fâcheusement manqué quand, en 1706, pour faciliter la navigation entre Saint-Etienne et Roanne, les passages les plus étroits des gorges de la Loire furent élargis. Il fut vite reconnu que ce fut la cause des grandes crues qui suivirent, de 1707 à 1710 . Il fallut donc, en 1711, construire trois digues pour compenser l'action modératrice des rochers disparus. La réaction a été rapide mais, néanmoins, d'effet limité à tel point que le gouvernement royal jugea bon de surélever et de renforcer les levées dans le Val de Loire. Les levées elles-mêmes sont exemples de ces aménagements dont l'intrusion dans le paysage est définitive même quand leur raison d'être devient douteuse. Ainsi que l'écrit Roger Dıon, « les événements (de 1733) montraient une fois de plus que le problème de la sécurité du Val était encore loin de sa solution. Mais il n'était plus permis d'en reprendre l'étude par la base : le système des levées était un fait qu'il fallait accepter et, depuis longtemps déjà, les discussions dont il était l'objet ne pouvaient plus porter que sur le choix des correctifs les plus opportuns ". Il est des aménagements irréversibles.

Le temps qui passe et les progrès de la connaissance et des techniques peuvent non seulement priver de leurs premiers effets bénéfiques des aménagements anciens mais révéler des effets nouveaux indésirables, mais qu'il faut corriger par de nouveaux travaux.

Les bras de rivières enserrant tout ou partie de maintes villes selon un bon plan de défense médiéval peuvent être fort contraignants pour les développements urbains postérieurs mais ils peuvent aussi, des siècles plus tard, rendre possible la création de plans d'eau si appréciés des citadins contemporains (Le Quesnoy, Bergues...). Comblés, les fossés peuvent aussi offrir des espaces opportuns à la croissance urbaine.

Des formes toutes nouvelles de tourisme contemporain ont rendu vie à des voies d'eau dont les équipements désuets, écluses, chemins de halage, anneaux d'amarrage, étaient devenus sans emploi. Telle société anglaise ayant son siège dans le département du Cher a multiplié par dix en dix ans sa flotte de bateaux qui sont donnés en location pour naviguer sur le canal latéral à la Loire et autres voies d'eau. Des entreprises semblables existent pour le bassin de la Maine, l'Alsace, la Lorraine, le Midi, la Bourgogne et la Bretagne. Les ingénieurs du $\mathrm{XvII}^{\mathrm{e}}$ siècle ne pouvaient certes pas prévoir que leurs ouvrages serviraient aux loisirs trois siècles plus tard et que l'ascenseur à péniches des Fontinettes deviendrait un monument historique.

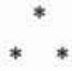

Les leçons tirées des ouvrages anciens équipant les rivières ont été bien comprises par des auteurs de programmes d'aménagement intégré, en cette seconde moitié du Xx ${ }^{\mathrm{e}}$ siècle. Ils savent que toutes leurs interventions sur les débits comme sur les lits des cours d'eau modifient des équilibres fragiles en constante évolution, à commencer par les profils longitudinaux et transversaux des vallées. Pour le cas où ils l'auraient oublié, le législateur a même prévu la description détaillée de ce que doit être l'étude d'impact. A une époque où les techniques évoluent et vieillissent très vite, on sait qu'il est prudent de prévoir la courte vie des aménagements aujourd'hui utiles et peut-être, dès demain, obsolètes. En conclusion, jamais l'homme n'a eu de si puissants moyens de dominer la nature et jamais pourtant il n'a dû prévoir de le faire avec plus de respect et de délicatesse. 


\section{Orientation bibliographique}

Bethemont J. - Le thème de l'eau dans la vallée du Rhône, essai sur la genèse d'un espace hydraulique. Saint-Etienne, 1972.

BRAVARD J.P. - Le Rhône, du Léman à Lyon. La Manufacture, Lyon, 1987.

DAChARRY M. - Hydrologie de la Loire en amont de Gien. 2 volumes, N.E.L., Paris, 1974.

Dessailly B. - L'aménagement du lit de la Têt à Perpignan. Bulletin de l'Association des Géographes Français, 1990, 1.

Dıon R. - Histoire des levées de la Loire. 10, rue Bénouville $\left(\mathrm{XVI}^{\mathrm{e}}\right)$, Paris, 1961.

GARNIER F.X.P. - Régime ou traité des rivières. 2 volumes, Paris, 1825.

Guillerme A. - Les temps de l'eau. La cité, l'eau et les techniques. Ed. du Champ Vallon, Seyssel, $2^{\mathrm{C}}$ édition, 1990.

IONIDES M.G. - The Regime of the Rivers Euphrate and Tigris. London, 1937.

... L'homme et l'eau au temps de la préhistoire. Actes du $112^{\circ}$ congrès national des Sociétés Savantes, Lyon, 1987. Editions du C.T.H.S., Paris, 1989.

... La ville et le fleuve. Actes du $112^{\circ}$ congrès national des Sociétés Savantes, Lyon, 1987, Editions du C.T.H.S., Paris, 1989.

... Dynamique et aménagement des lits fluviaux. Revue de Géographie de Lyon, $\mathrm{n}^{\circ} 4,1983$.

... Naviguer en Loire. Etudes ligériennes, 1986, n 22. 